\title{
Incidence, Predictors, and Impact on Survival of Left Ventricular Systolic Dysfunction and Recovery in Advanced Cancer Patients
}

\author{
Guilherme H. Oliveira, MD ${ }^{\mathrm{a}, *}$, Siddarth Mukerji, MD ${ }^{\mathrm{b}}$, Adrian V. Hernandez, MD ${ }^{\mathrm{c}, \mathrm{d}}$, \\ Marwan Y. Qattan, MD ${ }^{\mathrm{a}}$, Jose Banchs, $\mathrm{MD}^{\mathrm{e}}$, Jean-Bernard Durand, $\mathrm{MD}^{\mathrm{e}}$, Cezar Iliescu, $\mathrm{MD}^{\mathrm{e}}$, \\ Juan Carlos Plana, $\mathrm{MD}^{\mathrm{f}}$, and W.H. Wilson Tang, $\mathrm{MD}^{\mathrm{f}}$
}

\begin{abstract}
Although left ventricular (LV) dysfunction occurs not uncommonly in the course of cancer therapy, little is known about its natural history and prognostic impact on patients. To investigate the incidence, predictors, and impact on survival of LV systolic dysfunction and recovery during cancer therapy, we conducted a retrospective cohort observational study over 1 year at the University of Texas MD Anderson Cancer Center. We enrolled patients with a decrease in ejection fraction by echocardiography to $<50 \%$ while undergoing cancer therapy from January 2009 to December 2009. We collected and analyzed their chart data. Of 7,648 patients with echocardiograms in 2009, $366(4.8 \%)$ had ejection fraction $<50 \%$ and 104 met study criteria. LV systolic dysfunction was associated with cardiotoxic therapy in 53 patients (51\%). Recovery occurred in 57 patients (55\%) and was independently predicted by younger age, smaller left atrial volume index, and lower B-type natriuretic peptide. At last follow-up, 69 patients $(66 \%)$ were dead, and $35(34 \%)$ were alive. There was a $20 \%$ advantage in 2 -year survival among patients with LV systolic recovery compared with those without (95\% confidence interval $4 \%$ to $41 \%, p=0.02$ ). In this retrospective study, LV systolic dysfunction recovery occurred in over half of the patients, appeared independent of cardiotoxic etiology, and associated with a $20 \%$ survival benefit at 2 years. Multivariable predictors of recovery are younger age, a small left atrial volume index, and lower B-type natriuretic peptide. () 2014 Elsevier Inc. All rights reserved. (Am J Cardiol 2014;113:1893-1898)
\end{abstract}

In the noncancer population, myocardial recovery has been frequently observed in select cardiomyopathies, such as tachyarrhythmia induced, ${ }^{1}$ endocrine, ${ }^{2}$ nutritional, ${ }^{3}$ viral, ${ }^{4}$ catecholamine induced, ${ }^{5}$ and in patients with heart failure (HF) treated with mechanical circulatory support. ${ }^{6}$ Multiple predictors of myocardial recovery have been identified in noncancer patients with new onset left ventricular (LV) systolic dysfunction, such as LV end-diastolic volume, baseline LV ejection fraction (EF), and systolic blood pressure. $^{7}$ In contrast, LV systolic dysfunction in patients with cancer has mostly been studied from the prism of direct cytotoxic effects of cardiotoxic chemotherapy and thus, recovery has been shown to occur less often. ${ }^{8,9}$

Published data suggest that angiotensin-converting enzyme inhibitors (ACEIs) and $\beta$ blocker therapy and earlier

\footnotetext{
${ }^{\mathrm{a}}$ Division of Cardiovascular Medicine, Harrington Heart and Vascular Institute, University Hospitals Case Medical Center, Cleveland, Ohio; ${ }^{\mathrm{b}}$ Division of Cardiovascular Medicine, Department of Medicine, University of Michigan, Ann Arbor, Michigan; ${ }^{c}$ Department of Quantitative Health Sciences, Lerner Research Institute, Cleveland Clinic, Cleveland, Ohio; ${ }^{\mathrm{d}}$ Departamento de Cardiologia, Universidad Peruana de Ciencias Aplicadas, Lima, Peru; ' Division of Internal Medicine, Department of Cardiology, University of Texas MD Anderson Cancer Center, Houston, Texas; and ${ }^{\mathrm{f}}$ Department of Cardiovascular Medicine, Heart and Vascular Institute, Cleveland Clinic, Cleveland, Ohio. Manuscript received January 28, 2014; revised manuscript received and accepted March 5, 2014.

See page 1898 for disclosure information.

*Corresponding author: Tel: (216) 844-8242; fax: (216) 844-8318.

E-mail address: Guilherme.oliveira@uhhospitals.org (G.H. Oliveira).
}

intervention are associated with better chances of $\mathrm{LV}$ function recovery, ${ }^{10}$ although large-scale validation studies are lacking. The impact on survival of patients who recover from LV systolic dysfunction during cancer therapy has also not been fully studied. Herein, we sought to investigate the incidence, predictors, and impact on survival of recovery from LV systolic dysfunction in patients with cancer during cancer therapy.

\section{Methods}

With Institutional Review Board approval, we retrospectively queried the MD Anderson echocardiography laboratory database and identified sequential patients with echocardiograms performed during the year of 2009 whose EFs were $<50 \%$. We then excluded those without previous documentation of EF $>50 \%$ before initiation of cancer therapy or subsequent follow-up echocardiograms. The date of LV systolic dysfunction diagnosis was defined as that of the first abnormal echocardiogram in our system, subsequent to any imaging modality or documented office note recording a normal EF, which in many cases occurred before 2009. Patients in the cohort were in- or outpatients, age $\geq 18$ years with advanced cancer actively receiving cancer therapy. Patients were considered to have received cardiotoxic therapy if they had been treated with agents known to be associated with a $>5 \%$ risk of LV dysfunction at currently employed doses (anthracyclines and trastuzumab). ${ }^{11}$

All echocardiograms were reviewed by 2 independent investigators blinded to the sequence and dates of the echocardiograms, who re-measured all parameters according to the published guidelines of the American Society of 
Table 1

Univariable analysis: comparison of clinical characteristics between patients with and without recovery

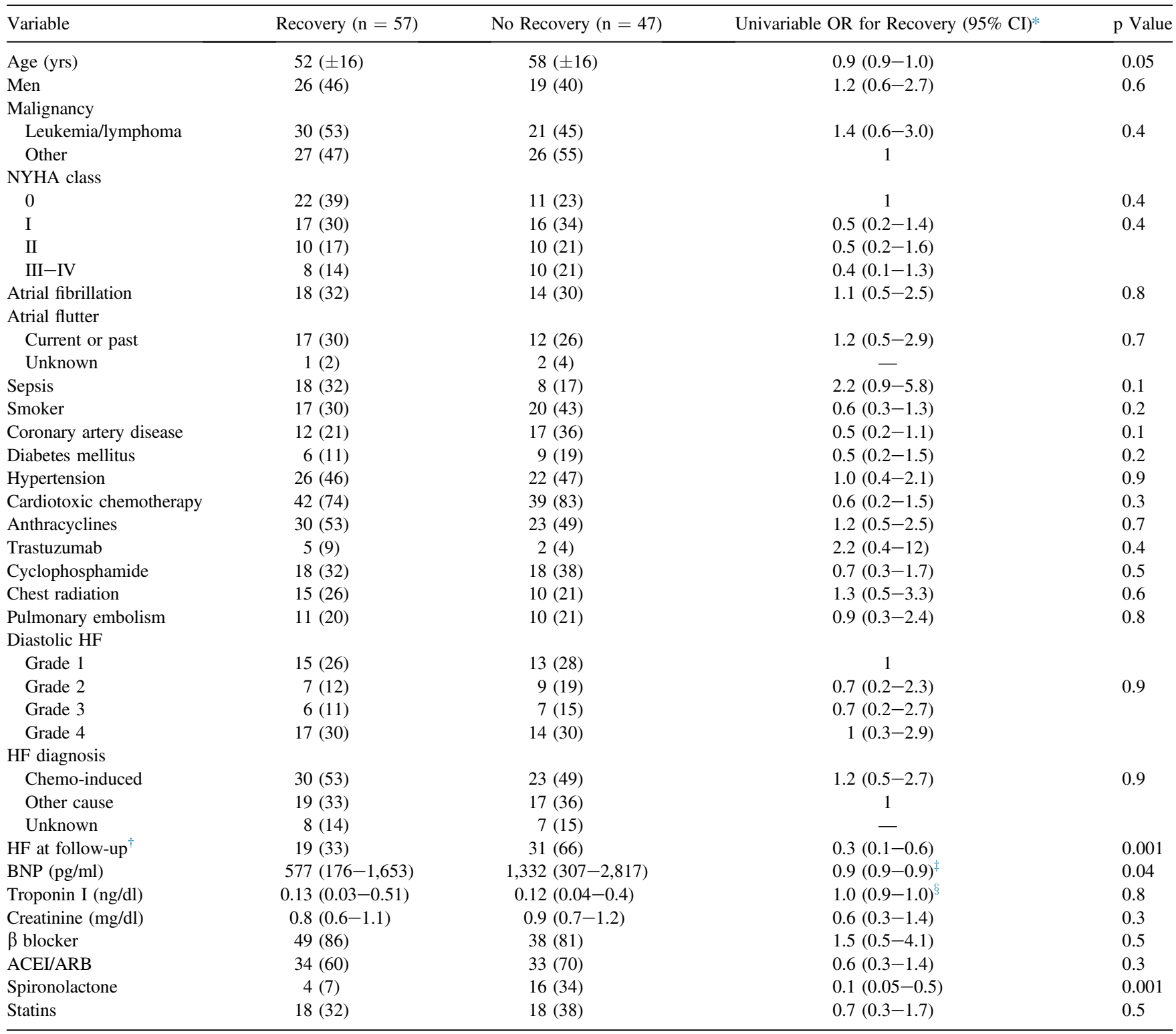

Data are presented as $\mathrm{n}(\%)$, mean $\pm \mathrm{SD}$, and median (IQR).

$\mathrm{ARB}=$ angiotensin receptor blocker; $\mathrm{IQR}=$ interquartile range; NYHA $=$ New York Heart Association; OR $=$ odds ratio.

* Per 1 unit increase of continuous variable.

${ }^{\dagger}$ Not considered for multivariable analysis as it was measured after baseline.

${ }^{\ddagger}$ Per 100 units increase.

${ }^{\S}$ Per 0.1 unit increase.

Echocardiography. ${ }^{12-14}$ We measured LVEF using the biplane method of disks (modified Simpson's). We included those with EF confirmed as $<50 \%$ and with previous EF $>50 \%$ by $\geq 5$ percentage points. All subsequent echocardiograms were similarly measured. Any discordance between the readers was resolved by consensus. Recovery was based on the last echocardiogram.

Echocardiographic 2-dimensional parameters including LVEF, left atrial volume index (LAVI), LV hypertrophy, LV end-diastolic dimension and volume, LV mass and LV mass index, and the presence of valvular heart disease were measured according to the published recommendations ${ }^{14}$ and documented. Valvular heart disease was subclassified into mild, moderate, or severe whether regurgitant or stenotic lesions were present. Diastolic function was assessed using Doppler and tissue Doppler techniques as published by the American Society of Echocardiography ${ }^{15}$ and classified as grade 1 (normal), 2 (impaired relaxation), 3 (pseudonormal), and 4 (restrictive).

LV systolic dysfunction recovery was defined as an increase in $\mathrm{EF}$ of $\geq 10 \%$ points from the lowest documented $\mathrm{EF}$ and absent if EF did not increase by $\geq 10 \%$ points. We also separately analyzed recovery defined as EF that returned to 
Table 2

Echocardiographic comparison between patients with and without left ventricular (LV) systolic function recovery

\begin{tabular}{|c|c|c|c|c|c|}
\hline Echocardiographic & \multicolumn{2}{|c|}{ EF Recovery } & $\mathrm{OR}^{*}$ & $\mathrm{CI}^{*}$ & $\mathrm{p}$ Value \\
\hline Ejection fraction at baseline & $34 \pm 8.5$ & $33 \pm 8.5$ & 1.01 & $0.97-1.06$ & 0.6 \\
\hline LV end-diastolic volume $\left(\mathrm{mm}^{3}\right)$ & $105 \pm 34$ & $114.5 \pm 39$ & 0.99 & $0.98-1.00$ & 0.2 \\
\hline LV mass index $\left(\mathrm{g} / \mathrm{m}^{2}\right)$ & $100 \pm 28$ & $109 \pm 36.5$ & 0.99 & $0.98-1.00$ & 0.1 \\
\hline \multicolumn{6}{|l|}{ LV hypertrophy } \\
\hline \multicolumn{6}{|l|}{ Pulmonary hypertension } \\
\hline No & $25(45)$ & $16(35)$ & 1.0 & & \\
\hline Mild & $32(38)$ & $13(28)$ & 1.0 & $0.4-2.6$ & 0.2 \\
\hline Moderate-to-severe & $10(18)$ & $17(37)$ & 0.4 & $0.1-1.1$ & \\
\hline
\end{tabular}

Data are presented as $\mathrm{n}(\%)$ and mean $\pm \mathrm{SD}$.

$\mathrm{OR}=$ odds ratio.

* Per 1 unit increase of continuous variable.

Table 3

Multivariable logistic model for left ventricular (LV) recovery

\begin{tabular}{lccc}
\hline Variable & OR $^{*}$ & CI $^{*}$ & p Value \\
\hline Age (yrs) & 0.95 & $0.92-0.99$ & 0.01 \\
Cardiotoxic chemotherapy & 0.40 & $0.10-1.30$ & 0.10 \\
Ejection fraction at baseline & 0.99 & $0.92-1.06$ & 0.70 \\
Left atrial volume index & 0.94 & $0.88-0.99$ & 0.04 \\
BNP & 0.97 & $0.94-0.99$ & 0.04 \\
LV end-diastolic dimension at baseline & 1.00 & $0.98-1.01$ & 0.60 \\
\hline
\end{tabular}

$\mathrm{OR}=$ odds ratio.

* Per 1 unit increase of continuous variable.

a value $\geq 50 \%$. We further subclassified recovery into 3 groups: (1) full recovery-defined as EF that increased $\geq 10 \%$ points and was $\geq 50 \%$ at the last echocardiogram; (2) partial recovery-defined as EF that increased by $\geq 10 \%$ points but remained $<50 \%$; and (3) no recovery-defined as $<10 \%$ point increase in $\mathrm{EF}$ and $\mathrm{EF}<50 \%$.

Clinical data were retrospectively collected from the time of diagnosis of LV systolic dysfunction, with special attention to New York Heart Association class and HF symptoms, presence of atrial fibrillation, hemodynamic shock (defined as low blood pressure requiring admission to intensive care unit and specific therapy for hypotension), history of any coronary artery disease, diabetes mellitus, hypertension, and tobacco use. Pulmonary hypertension was defined as mean pulmonary artery pressure $>25 \mathrm{~mm} \mathrm{Hg}$ at rest of $>30 \mathrm{~mm} \mathrm{Hg}$ at exercise. Cancer therapy at time of LV systolic dysfunction was also documented, with specific attention to use of chemotherapy agents and radiation therapy to the chest. Laboratory data were documented when obtained at the time of LV systolic dysfunction, specifically B-type natriuretic peptide (BNP) levels, serum troponin-I levels, and serum creatinine. Additionally, attempts at determination of etiology of HF were also investigated and documented.

HF therapy instituted at the time of LV systolic dysfunction was documented according to the major family of agents, that is ACEIs, $\beta$ blockers, diuretics, aldosterone receptor antagonist, and so on. Patients were treated according to the published guidelines ${ }^{16,17}$ to the extent that hemodynamic status and concomitant cancer therapies permitted. Follow-up was included retrospectively from the date of LV systolic dysfunction diagnosis until November 2011. Date and cause of death were obtained from the medical records. For those alive by November 2011, clinical status was documented.

Categorical variables are expressed as number and percent and continuous variables as mean (SD or median [interquartile range]) when the distribution was not normal. Comparisons between patients with and without recovery were done with the Fisher's exact test for categorical variables and the Wilcoxon rank-sum test for continuous variables. Potential predictors of recovery were those available at baseline. Univariable logistic regression analyses were performed to evaluate the association between a patient variable and a recovery. The association was expressed as odds ratio and its $95 \%$ confidence interval $(\mathrm{CI})$. Variables with a $\mathrm{p} \leq 0.2$ in univariable analysis and some prespecified variables (baseline LVEF, LV end-diastolic dimension, and the use of cardiotoxic chemotherapy) were chosen for multivariable logistic regression analysis, where variables with a $p<0.05$ were considered independently associated with recovery. Survival was described with the KaplanMeier method, and differences between patients with and without recovery were tested with the log-rank test. To evaluate the discriminative ability of LAVI to differentiate between recovery and no recovery, we performed receiveroperating characteristic analysis and quantified the ability with the c-statistic. SAS 9.2 (SAS Institute Inc., Cary, North Carolina) was used for all statistical analyses.

\section{Results}

Throughout 2009, of 7,648 consecutive patients with cancer who underwent echocardiographic evaluation, 366 (4.8\%) had EF $<50 \%$. Of these, 104 patients had previously documented normal EF and at least 1 subsequent echocardiogram, meeting inclusion criteria. Baseline characteristics are listed in Table 1. 


\section{Overall Cohort Survival}

Product-limit Survival Estimate with Numbers of Subjects at Risk

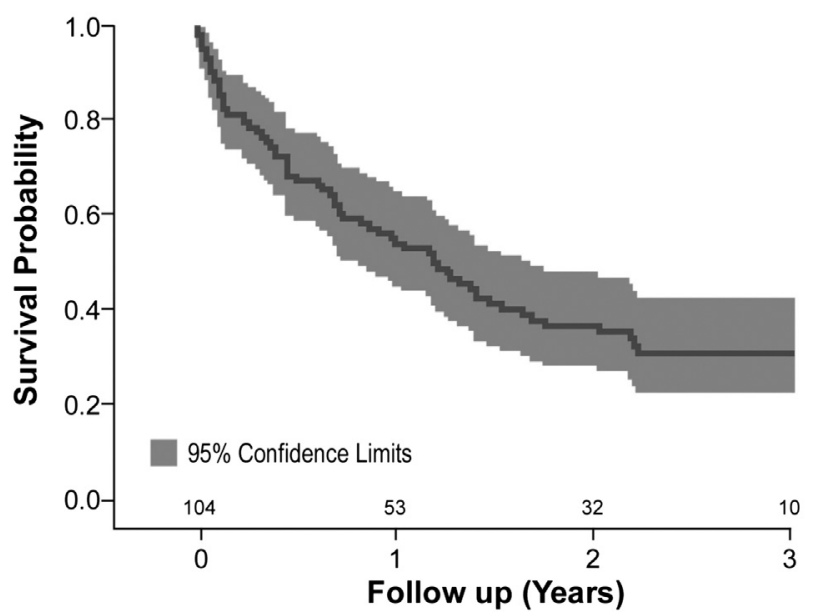

Figure 1. Overall cohort survival: product-limit 3-year survival estimate with number of subjects at risk.

The most common malignancies were leukemia (30\%), lymphoma (19\%), genitourinary cancer $(11 \%)$, breast (10\%), lung (10\%), sarcomas (6\%), and others (17\%). In this cohort, $32 \%$ and $68 \%$ of patients had stage III and IV cancer, respectively.

Cancer therapy consisted of anthracyclines, $48 \%$; cyclophosphamide, 35\%; vascular endothelial growth factor inhibitors, $26 \%$; and trastuzumab, $7 \%$. In this cohort, 25 patients (24\%) received radiation therapy to the chest. Cardiotoxic therapy had been prescribed in 60 patients (58\%).

The mean documented EF before chemotherapy initiation was $61 \% \pm 9 \%$. Regional wall motion abnormalities were described in 41 patients $(40 \%)$. Information on diastolic function was available in 91 patients $(88 \%)$ and was abnormal in 73 patients $(80 \%)$, with 22 patients $(30 \%)$ presenting with Grade 4 diastolic dysfunction. Echocardiographic parameters at presentation of LV systolic dysfunction of patients with and without recovery are listed in Table 2.

LV systolic dysfunction was thought to be chemotherapy induced in 53 patients (51\%), other causes such as sepsis and arrhythmia in 36 patients (35\%), and unknown in 15 patients (14\%). Evaluation of coronary artery disease was carried out in 38 patients (35\%), none of whom had severe enough coronary artery disease to account for their cardiomyopathy.

At the last echo, LV systolic dysfunction recovery had occurred in 57 patients $(55 \%)$. The mean EF of those with recovery versus no recovery was $51 \% \pm 0.8 \%$ and $31 \% \pm$ $0.9 \%$ ( $<<0.0001$ ), respectively. In the recovery group, LVEF was $>55 \%$ in 20 patients (35\%), 50\% to $54 \%$ in 13 patients (23\%), $45 \%$ to $49 \%$ in 15 patients (26\%), and $40 \%$ to $44 \%$ in 9 patients $(16 \%)$.

Baseline characteristics of recovered versus not were similar (Table 1), except for LAVI that was significantly lower in those who recovered $\left(26 \pm 8\right.$ vs $33 \pm 10 \mathrm{ml} / \mathrm{m}^{2}$, $\mathrm{p}=0.0002$ ). HF treatment was administered to 102 patients $(98 \%)$. Interestingly, there was a significantly higher use of spironolactone in those who did not recover compared with

\section{Survival of Patients with and without Recovery of LV Function}

Product-limit Survival Estimate with Numbers of Subjects at Risk

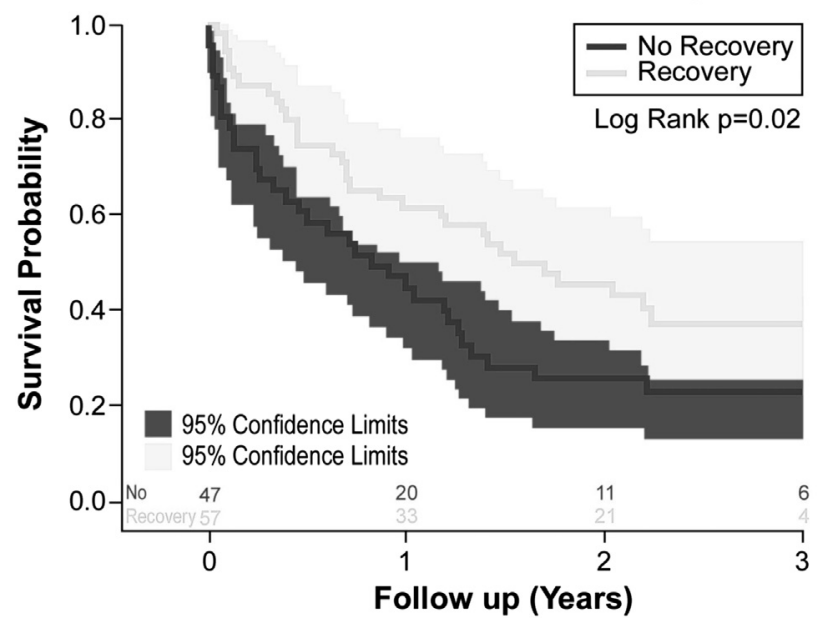

Figure 2. Survival of patients with and without the recovery of LV function: product-limit survival estimate with numbers of subjects at risk demonstrating favorable survival of patients who experience LV function recovery.

those who did (35\% vs $7 \%$, respectively, $\mathrm{p}=0.0004)$. Finally, there was no difference in rates of recovery between those with exposure to cardiotoxic therapy and those with other etiologies of LV systolic dysfunction ( $61 \%$ vs $38.5 \%$, respectively, odds ratio $1.4,95 \% \mathrm{CI} 0.64$ to $3.06, \mathrm{p}=0.39$ ).

When we analyzed recovery as return of LV function to $\mathrm{EF} \geq 50 \%, 33$ patients (32\%) recovered and 71 patients $(68 \%)$ did not. Further, when stratified into full (EF increase $\geq 10 \%$ points and $\mathrm{EF} \geq 50 \%$ ), partial (EF increase $\geq 10 \%$ points and $\mathrm{EF}<50 \%$ ), and no recovery (EF increase $<10 \%$ and points and $\mathrm{EF}<50 \%)$, there were $33(32 \%), 24(23 \%)$, and $47(45 \%)$ patients, respectively.

We conducted uni- and multivariable analyses to determine baseline predictors of recovery. Results of the univariable analysis are listed in Tables 1 and 2. Multivariable analysis confirmed age, LAVI, and BNP as independent predictors of recovery (Table 3 ).

A receiver-operating characteristic analysis for LAVI showed an area under the curve of 0.69 (95\% CI 0.55 to 0.79 ) suggesting a modest discriminatory power of LAVI for predicting recovery. Assuming that higher values of LAVI are associated with lower chances of recovery, a LAVI of $30 \mathrm{ml} / \mathrm{m}^{2}$ was able to discriminate between recovery versus no recovery with a sensitivity of $70 \%$ and a specificity of $66 \%$.

The mean follow-up was $1.94 \pm 1.6$ years; at last followup, 69 patients $(66 \%)$ were dead. Survival of the entire cohort is shown in Figure 1. When stratified by the recovery of LV function, there was significant survival advantage in patients with LV function recovery (Figure 2). At 1,2, and 3 years, differences in survival between patients with and without recovery were $15 \%, 20 \%$, and $14 \%(\mathrm{p}=0.02,95 \%$ CI $1 \%$ to $41 \%$ ), respectively. Finally, when we stratified the groups as full recovery (EF increase $\geq 10 \%$ and points and $\mathrm{EF} \geq 50 \%$ ), partial recovery (EF increase $\geq 10 \%$ and points and $\mathrm{EF}<50 \%$ ), and no recovery (EF increase $<10 \%$ and points and $\mathrm{EF}<50 \%$ ), there was a survival advantage to 


\section{Survival of Patients with Full,} Partial and No Recovery

Product-limit Survival Estimates with Numbers of Subjects at Risk

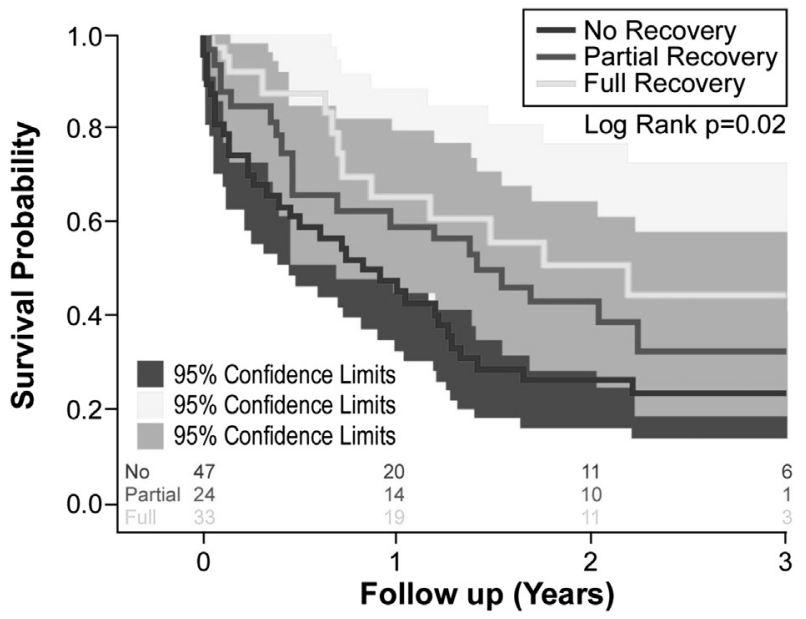

Figure 3. Survival of patients with full, partial, and no recovery of LV function: product-limit survival estimate with numbers at risk, demonstrating favorable survival of patients with full or partial recovery compared with those without recovery of LV function.

those with partial or full recovery compared with those without (Figure 3). Among the 69 patients who died by November 2011,33 patients $(48 \%)$ died of cancer progression, 29 patients $(42 \%)$ died of treatment-related complications, 25 patients (24\%) died of sepsis, 4 patients (4\%) died of intracranial hemorrhage, 1 patient (1\%) died of HF, and 6 patients (9\%) unknown.

\section{Discussion}

We report herein the largest cohort of patients with cancer who developed LV systolic dysfunction during cancer therapy. We also illustrate the incidence and determinants of recovery from LV systolic dysfunction, and most importantly, its impact on survival. Our main finding is that recovery from LV systolic dysfunction in patients with advanced cancer occurs not infrequently and results in prolonged survival. An interesting finding is that LV systolic dysfunction occurred independently of cardiotoxic therapy in almost a third of patients and did not appear to significantly impact the likelihood of recovery. Importantly, we show that recovery can be predicted by a smaller LAVI, lower BNP, and younger age at presentation.

There are several interesting observations in our cohort that are perhaps distinctively different from previous reports as we approach the problem from the perspective of LV systolic dysfunction detection in a dedicated cancer hospital in which all patients have advanced cancer and are undergoing a wide range of cancer therapies. In this mixed population of patients with stages III and IV cancer, LV systolic dysfunction occurred in $<5 \%$ of patients, perhaps in part because of insufficient testing with echocardiography. Most patients presented with asymptomatic LV systolic dysfunction, preserved LV end-diastolic volume, and only a minority with New York Heart Association class IV symptoms.

Importantly, almost half of the patients who developed LV systolic dysfunction had not received therapy considered significantly cardiotoxic, suggesting that half of the patients had other causes for developing LV systolic dysfunction. These patients more likely developed LV systolic dysfunction from acute illnesses, tachycardia, and myocarditis, whether inflammatory, cytokine induced, or viral induced. This finding underscores the need for appropriate etiologic investigation of LV systolic dysfunction and not to automatically assume that LV systolic dysfunction in patients with cancer is caused by chemotherapeutic agents (guilty by association).

Recovery from LV systolic dysfunction occurred in over half of the patients, independent of cardiotoxic drug use. This finding corroborates the notion that etiologically, LV systolic dysfunction in advanced patients with cancer most often resembles that of patients with myocarditis and acute cardiomyopathies, ${ }^{7,18}$ where the incidence of myocardial recovery is high. Our rates of recovery are different previous studies that focused on patients who had received cardiotoxic chemotherapy agents (e.g., doxorubicin), where the assumption was that the agent was always responsible for the cardiomyopathy. Although that may be true in some cases, the finding that a subset of patients developed LV systolic dysfunction without receiving cardiotoxic therapy challenges this assumption.

Also, contrary to previous reports, ${ }^{10}$ recovery did not appear to be determined by the usage of HF medications, perhaps because of the high percentages of $\beta$ blocker and ACEIs in both groups. Interestingly, the use of aldosterone antagonists was inversely associated with LV systolic dysfunction recovery. Although the numbers are small, this finding may be explained by that only patients who did not initially respond to ACEIs and $\beta$ blocker therapy were treated with these agents.

Importantly, we also show that younger age, lower BNP, and smaller LAVI independently predict recovery. A smaller LAVI at the time of LV systolic dysfunction presentation was a strong independent predictor of subsequent recovery among patients with cancer with LV systolic dysfunction. LAVI has been described as an important prognostic marker of cardiovascular disease, ${ }^{19}$ but this is the first time it is linked to improvement of LV function in patients with LV systolic dysfunction. It is possible that a larger LAVI at presentation is an early marker for patients who have high filling pressures, who have rapidly progressive remodeling, and who will eventually progress to dilated $\mathrm{LV}$, from which they are less likely to recover. The finding that lower BNP levels were also independently associated with recovery corroborates this hypothesis. Further supporting this argument is that, unlike previously described, ${ }^{7} \mathrm{LV}$ end-diastolic volume was not a determinant of LV recovery. The reason for this may be that the predominance of diastolic dysfunction from either cardiotoxicity or radiation therapy may have contributed to increased LAVI without LV dilatation. Finally, this may support the anecdotal observation that, unlike in other forms of cardiomyopathy, LV dilatation appears to be a less prominent feature in patients with chemotherapy-induced cardiomyopathy that progress to end-stage HF. ${ }^{20}$

Our patients had very poor survival, reflecting the adverse prognosis associated with stage III or IV cancer, ${ }^{21}$ and that mortality was driven by cancer progression and treatment-related complications. Nevertheless, recovery of 
LV function was associated with a $20 \%$ increase in 2-year survival. It appears, therefore, that despite the severity of their underlying disease, there is clearly an advantage to recovering LV function. It is possible that patients with better LV function were treated more aggressively, or without interruptions, resulting in prolonged progression free and overall survival. Taken together, our findings underscore the need for careful monitoring of LV function in patients with cancer, with early detection and aggressive treatment of LV systolic dysfunction.

Major limitations to this study are selection and referral biases, unavoidable because all patients were recruited from the echocardiography database and were a priori found to have an abnormal EF, presumably because a cardiac abnormality was suspected. Because the timing and doses of chemotherapy are not documented, a definitive temporal correlation with LV systolic dysfunction cannot be made, limiting the ability to establish causal relation in regard to LV systolic dysfunction etiology. Finally, although this is the largest series available for this type of patients, the numbers remain relatively low and therefore prone to type 2 error.

\section{Disclosure}

The authors have no conflicts of interest to disclose.

1. Packer DL, Bardy GH, Worley SJ, Smith MS, Cobb FR, Coleman RE, Gallagher JJ, German LD. Tachycardia-induced cardiomyopathy: a reversible form of left ventricular dysfunction. Am J Cardiol 1986;57: 563-570.

2. Sachs RN, Valensi P. Reversible cardiomyopathy due to thyrotoxicosis. Am J Cardiol 1993;71:501.

3. Larner AJ. Selenium deficiency, reversible cardiomyopathy and shortterm intravenous feeding. Postgrad Med J 1994;70:764-765.

4. Barbandi M, Cordero-Reyes A, Orrego C, Torre-Amione G, Seethamraju H, Estep J. A case series of reversible acute cardiomyopathy associated with H1N1 influenza infection. Methodist Debakey Cardiovasc $J$ 2012;8:42-45.

5. Wittstein IS, Thiemann DR, Lima JA, Baughman KL, Schulman SP, Gerstenblith G, Wu KC, Rade JJ, Bivalacqua TJ, Champion HC. Neurohumoral features of myocardial stunning due to sudden emotional stress. N Engl J Med 2005;352:539-548.

6. Boehmer JP, Starling RC, Cooper LT, Torre-Amione G, Wittstein I, Dec GW, Markham DW, Zucker MJ, Gorcsan J 3rd, McTiernan C, Kip K, McNamara DM. Left ventricular assist device support and myocardial recovery in recent onset cardiomyopathy. J Card Fail 2012;18:755-761.

7. McNamara DM, Starling RC, Cooper LT, Boehmer JP, Mather PJ, Janosko KM, Gorcsan J 3rd, Kip KE, Dec GW. Clinical and demographic predictors of outcomes in recent onset dilated cardiomyopathy: results of the IMAC (Intervention in Myocarditis and Acute Cardiomyopathy)-2 study. J Am Coll Cardiol 2011;58:1112-1118.

8. Fazio $\mathrm{S}$, Palmieri EA, Ferravante $\mathrm{B}$, Bonè $\mathrm{F}$, Biondi $\mathrm{B}$, Saccà L. Doxorubicin-induced cardiomyopathy treated with carvedilol. Clin Cardiol 1998:21:777-779.

9. Yusuf SW, Ilias-Khan NA, Durand JB. Chemotherapy-induced cardiomyopathy. Expert Rev Cardiovasc Ther 2011;9:231-243.

10. Cardinale D, Colombo A, Lamantia G, Colombo N, Civelli M, De Giacomi G, Rubino M, Veglia F, Fiorentini C, Cipolla CM.
Anthracycline-induced cardiomyopathy: clinical relevance and response to pharmacologic therapy. JAm Coll Cardiol 2010;55:213-220.

11. Yeh ET, Bickford CL. Cardiovascular complications of cancer therapy: incidence, pathogenesis, diagnosis, and management. J Am Coll Cardiol 2009;53:2231-2247.

12. Douglas PS, DeCara JM, Devereux RB, Duckworth S, Gardin JM, Jaber WA, Morehead AJ, Oh JK, Picard MH, Solomon SD, Wei K, Weissman NJ. Echocardiographic imaging in clinical trials: American Society of Echocardiography Standards for echocardiography core laboratories: endorsed by the American College of Cardiology Foundation. J Am Soc Echocardiogr 2009;22:755-765.

13. Reeves ST, Glas KE, Eltzschig H, Mathew JP, Rubenson DS, Hartman GS, Shernan SK. Guidelines for performing a comprehensive epicardial echocardiography examination: recommendations of the American Society of Echocardiography and the Society of Cardiovascular Anesthesiologists. J Am Soc Echocardiogr 2007:20:427-437.

14. Lang RM, Bierig M, Devereux RB, Flachskampf FA, Foster E, Pellikka PA, Picard MH, Roman MJ, Seward J, Shanewise JS, Solomon SD, Spencer KT, Sutton MS, Stewart WJ. Recommendations for chamber quantification: a report from the American Society of Echocardiography's Guidelines and Standards Committee and the Chamber Quantification Writing Group, developed in conjunction with the European Association of Echocardiography, a branch of the European Society of Cardiology. J Am Soc Echocardiogr 2005;18: $1440-1463$.

15. Dokainish H, Nguyen JS, Bobek J, Goswami R, Lakkis NM. Assessment of the American Society of Echocardiography- European Association of Echocardiography guidelines for diastolic function in patients with depressed ejection fraction: an echocardiographic and invasive haemodynamic study. Eur J Echocardiogr 2011;12: 857-864.

16. Jessup M, Abraham W, Casey D, Feldman A, Francis G, Ganiats T, Konstam M, Mancini D, Rahko P, Silver M, Stevenson L, Yancy C. 2009 focused update: ACCF/AHA guidelines for the diagnosis and management of heart failure in adults: a report of the american College of Cardiology Foundation/American Heart Association Task Force on Practice guidelines: developed in collaboration with the International Society for Heart and Lung Transplantation. Circulation 2009;119: 1977-2016.

17. Hunt SA, Abraham WT, Chin MH, Feldman AM, Francis GS, Ganiats TG, Jessup M, Konstam MA, Mancini DM, Michl K, Oates JA, Rahko PS, Silver MA, Stevenson LW, Yancy CW. 2009 focused update incorporated into the ACC/AHA 2005 Guidelines for the Diagnosis and Management of Heart Failure in Adults: a report of the American College of Cardiology Foundation/American Heart Association Task Force on Practice Guidelines: developed in collaboration with the International Society for Heart and Lung Transplantation. Circulation 2009;119:e391-e479.

18. Teeter WA, Thibodeau JT, Rao K, Brickner ME, Toto KH, Nelson LL, Mishkin JD, Ayers CR, Miller JG, Mammen PP, Patel PC, Markham DW, Drazner MH. The natural history of new-onset heart failure with a severely depressed left ventricular ejection fraction: implications for timing of implantable cardioverter-defibrillator implantation. Am Heart J 2012;164:358-364.

19. Abhayaratna WP, Seward JB, Appleton CP, Douglas PS, Oh JK, Tajik AJ, Tsang TS. Left atrial size: physiologic determinants and clinical applications. J Am Coll Cardiol 2006;47:2357-2363.

20. Oliveira GH, Hardaway BW, Kucheryavaya AY, Stehlik J, Edwards LB, Taylor DO. Characteristics and survival of patients with chemotherapyinduced cardiomyopathy undergoing heart transplantation. J Heart Lung Transpl 2012;31:805-810.

21. NSCLC Meta-Analyses Collaborative Group. Chemotherapy in addition to supportive care improves survival in advanced non- small-cell lung cancer: a systematic review and meta-analysis of individual patient data from 16 randomized controlled trials. J Clin Oncol 2008;26:4617-4625. 